\title{
An Integration Strategy based on Fuzzy Clustering and Level Set Method for Cell Image Segmentation
}

\author{
Amin Gharipour \\ School of Information and Com munication Technology \\ Gold Coast campus, Griffith University, QLD4222, \\ Australia \\ amin.gharipour@griffithuni.edu.au
}

\author{
Alan Wee-Chung Liew \\ School of Information and Communication Technology \\ Gold Coast campus, Griffith University, QLD4222, \\ Australia \\ a.liew@griffith.edu.au
}

\begin{abstract}
In this study a new image segmentation framework which combines the Fuzzy c means clustering and the level set method is presented. Using this framework, the well-known Chan and Vese's level set technique and classical Bayes classifier are employed to obtain a prior membership value for each pixel based on region information. Next, a novel clustering model based on fuzzy c-mean clustering assisted by prior membership values is used to obtain the final segmentation. Experiments performed on highthroughput fluorescence microscopy colon cancer cell images, which are commonly utilized for the study of many normal and neoplastic procedures, indicate a significant improvement in accuracy when compared to several existing techniques.
\end{abstract}

Index Terms-Cell image segmentation; fuzzy c-means; Chan-Vese method; level set; classical Bayes classifier

\section{INTRODUCTION}

Computer-based Cell nucleus segmentation is one of the critical tasks in the quantification of protein expression and analyzing the function of cells in fluorescence microscopy images. In general, Image segmentation methods based on fuzzy clustering and level set methods are two categories of important techniques for partitioning images into different non-overlapping regions. Whereas level set methods impose the constraints on boundary smoothness, fuzzy clustering utilizes spectral properties of image pixels in performing segmentation.

An initial formulation of level set methods was presented by Malladi et al. [1] and Caselles et al. [2]. Level set techniques extract contours as the zero level set of a function formulated in a higher dimension space based on dynamic implied boundaries and partial differential equations (PDEs) [3]. Chan and Vese [4] proposed a level set segmentation method based on an active contour model using the energy minimization method and incorporating region-based information in the energy functional as an extra constraint.

As a famous unsupervised clustering technique, fuzzy c-means (FCM) is based on the idea of uncertainty of belonging described by a membership grade that falls between zero to one and has the ability to employ more information from the image in comparison with the crisp or hard segmentation methods. FCM reveals the original structure of the image data and segments it into parts with nearly constant spectral properties. An important disadvantage of FCM is that when it is utilized as a segmentation method in image processing and analysis, topological information and spatial relationship is totally ignored $[5,6]$.

In image segmentation, spectral properties of pixels and smoothness of the segmented boundary should be considered simultaneously. Motivated by this consideration, a three-step integration strategy based on Chan and Vese's (CV) method, classical Bayes classifier and FCM is proposed in this paper. The rest of this paper is organized as follows. The next section elaborates on $\mathrm{CV}$ segmentation and describes FCM. Section three presents a three-step integration strategy in detail. The experimental results based on high-throughput fluorescence microscopy images are considered in Section 4. Concluding remarks are drawn in Section 5.

\section{METHODS}

Before presenting the novel integration strategy, the FCM and $\mathrm{CV}$ methods are explained in this section and reviewed the important concepts to provide the required background for our hybrid algorithm. This commences with a remark on the CV level set method.

\section{A. Chan-Vese model}

The CV model is formulated based on the Mumford-Shah model [7] which can be written as follows:

$\mathrm{E}^{\mathrm{MS}}(\mathrm{I}, \mathrm{C})$

$=\int_{\Omega}\left|\mathrm{I}_{0}(\mathrm{x}, \mathrm{y})-\mathrm{I}(\mathrm{x}, \mathrm{y})\right|^{2} \mathrm{dxdy}+\mu \int_{\Omega \backslash \mathrm{C}}|\nabla \mathrm{I}(\mathrm{x}, \mathrm{y})|^{2} \mathrm{dxdy}$

$+\mathrm{v} .|\mathrm{C}|$

where $\Omega$ denotes the image domain, $C \subset \Omega$ denotes the closed, smooth segmenting curve, $\mathrm{I}_{0}: \Omega \rightarrow \mathrm{R}$ denotes a given image, I denotes the piecewise smooth approximation of $\mathrm{I}_{0}$ with discontinuities along $\mathrm{C},|\mathrm{C}|$ indicates the length of $\mathrm{C}, \mu$ and $\mathrm{v}$ are positive constants.

$\mathrm{CV}$ method solves the minimization of MumfordShah energy functional through minimizing the following energy functional: 
$\mathrm{E}^{\mathrm{CV}}\left(\mathrm{c}_{1}, \mathrm{c}_{2}, \mathrm{C}\right)=\lambda_{1} \cdot \int_{\text {inside(c) }}\left|\mathrm{I}_{0}(\mathrm{x}, \mathrm{y})-\mathrm{c}_{1}\right|^{2} \mathrm{dxdy}+$
$\lambda_{2} \cdot \int_{0}\left|\mathrm{I}_{0}(\mathrm{x}, \mathrm{y})-\mathrm{c}_{2}\right|^{2} \mathrm{dxdy}+\mu .|\mathrm{C}|$

where $\lambda_{1}, \lambda_{2}$ and $\mu$ are positive constants and $c_{1}, c_{2}$ are the mean intensities of $\mathrm{I}_{0}$ inside and outside the segmenting curve, respectively.

In the CV method, the level set method is used to find the minimum value of the functional (2). The curve $\mathrm{C}$ is replaced with $\varnothing(\mathrm{x}, \mathrm{y})$, called the level set function, such that:

$\left\{\begin{array}{l}\varnothing(\mathrm{x}, \mathrm{y})>0 \text { if }(\mathrm{x}, \mathrm{y}) \text { is inside } \mathrm{C} \\ \varnothing(\mathrm{x}, \mathrm{y})=0 \mathrm{if}(\mathrm{x}, \mathrm{y}) \text { is on } C \\ \varnothing(\mathrm{x}, \mathrm{y})<0 \text { if }(\mathrm{x}, \mathrm{y}) \text { is outside } \mathrm{C}\end{array}\right.$

Thus (2) can be modified as follows:

$\mathrm{E}^{\mathrm{CV}}\left(\mathrm{c}_{1}, \mathrm{c}_{2}, \varnothing(\mathrm{x}, \mathrm{y})\right)=$

$\lambda_{1} \cdot \int_{\Omega}\left|\mathrm{I}_{0}(\mathrm{x}, \mathrm{y})-\mathrm{c}_{1}\right|^{2} \mathrm{H}_{\varepsilon}(\varnothing(\mathrm{x}, \mathrm{y})) \mathrm{dxdy}+$

$\lambda_{2} \cdot \int_{\Omega}^{\Omega}\left|I_{0}(x, y)-c_{2}\right|^{2}\left(1-H_{\varepsilon}(\varnothing(x, y)) d x d y+\right.$

$\mu \cdot \int_{\Omega} \delta_{\varepsilon}(\varnothing(\mathrm{x}, \mathrm{y}))|\nabla \emptyset(\mathrm{x}, \mathrm{y})| \mathrm{dxd}$

where $\mathrm{H}_{\varepsilon}(\mathrm{z})$ and $\delta_{\varepsilon}(\mathrm{z})$ denotes the regularized forms of the Heaviside function $\mathrm{H}(\mathrm{z})$ and Dirac delta function $\delta(\mathrm{z})$ given by $H(z)=\left\{\begin{array}{l}1 \text { if } z \geq 0 \\ 0 \text { if } z \leq 0\end{array} \quad\right.$ and $\quad \delta(z)=\frac{\partial H(z)}{\partial z}$ respectively.

The existence of minimizer for (3) has been considered in $[7,8]$. Employing Euler- Lagrange method, the level set function $\varnothing(\mathrm{x}, \mathrm{y})$ can be updated as follows:

$\frac{\partial \emptyset}{\partial \mathrm{t}}=\delta_{\varepsilon}(\varnothing)\left[\mu \operatorname{div}\left(\frac{\nabla \emptyset}{|\nabla \varnothing|}\right)-\chi_{1}\left(\mathrm{I}_{0}-\mathrm{c}_{1}\right)^{2}+\chi_{2}\left(\mathrm{I}_{0}-\mathrm{c}_{2}\right)^{2}\right]$

At each iteration $c_{1}$ and $c_{2}$ are updated by:

$\mathrm{c}_{1}(\varnothing)=\frac{\int_{\Omega} \mathrm{I}_{0}(\mathrm{x}, \mathrm{y}) \mathrm{H}_{\varepsilon}(\varnothing(\mathrm{x}, \mathrm{y})) \mathrm{dxdy}}{\int_{\Omega} \mathrm{H}_{\varepsilon}(\varnothing(\mathrm{x}, \mathrm{y})) \mathrm{dxdy}}$

$c_{2}(\varnothing)=\frac{\int_{\Omega} I_{0}(x, y)\left(1-H_{\varepsilon}(\varnothing(x, y))\right) d x d y}{\int_{\Omega}\left(1-H_{\varepsilon}(\varnothing(x, y))\right) d x d y}$

\section{$B$. The fuzzy c-means clustering algorithm}

The fuzzy c-means clustering algorithm, as an extension of the well-known k-means clustering method, was first proposed by Dunn [9], and further developed by Bezdek [10]. The goal of FCM is to partition $\xi=\left(\xi_{1}, \ldots, \xi_{n}\right)$, i.e., a set of voxel or pixel locations in $\Omega$, into $\eta$ clusters, that are defined by prototypes $v=\left(v_{1}, \ldots, v_{\eta}\right)$. With fuzzy clustering each $\xi_{\mathrm{j}}$ is a member of all partitions simultaneously, but with different membership grades. The FCM algorithm achieves the final solution by solving the following minimization problem:

$\operatorname{Minimize~}_{\mathrm{FCM}}(\mathrm{U}, \mathrm{V})=\sum_{\mathrm{i}=1}^{\mathrm{n}} \sum_{\mathrm{j}=1}^{\mathrm{n}} \mathrm{u}_{\mathrm{ij}}^{\mathrm{m}}\left\|\xi_{\mathrm{j}}-\mathrm{v}_{\mathrm{i}}\right\|^{2}$

Subject to $\mathrm{U} \in \mathcal{M}$

where $\mathcal{M}=\left\{\mathrm{U}=\left[\mathrm{u}_{\mathrm{ij}}\right]_{\substack{\mathrm{i}=1, \ldots, n \\ \mathrm{j}=1, \ldots, \mathrm{n}}} \mid \mathrm{u}_{\mathrm{ij}} \in[0,1], \sum_{\mathrm{i}=1}^{\eta} \mathrm{u}_{\mathrm{ij}}=1\right\}$
The parameter $\mathrm{m}, 1 \leq \mathrm{m} \leq \infty$, affects the fuzziness of the clusters. As can be seen from (7) every pixel has the same weight in the image's data set. Fuzzy clustering under constraint (7) is regularly called probabilistic clustering and $\mathrm{u}_{\mathrm{ij}}$ can be considered as the posterior probability $\mathrm{p}\left(\eta_{\mathrm{i}} \mid \xi_{j}\right)[11]$. Alternating optimization, which alternates between optimizations of $\tilde{J}_{\mathrm{FCM}}(\mathrm{U} \mid \dot{\mathrm{V}})$ over $U$ with fixed $\dot{v}$ and $\tilde{\mathrm{J}}_{\mathrm{FCM}}(\mathrm{v} \mid \dot{U})$ over $\mathrm{v}$ with fixed $\dot{\mathrm{U}}$, converges to a local minimizer or a saddle point of $\mathrm{J}_{\mathrm{FCM}}[12]$.

\section{INTEGRATION STRATEGY}

The integration strategy consists of three steps (Fig. 1). In the first step, the segmentation based on region information is performed using the $\mathrm{CV}$ model. Classical Bayes classifier is used to estimate prior membership degrees using CV's segmentation results in the second step. Finally, the novel supervised FCM is presented to utilize prior membership degrees given by $\mathrm{CV}$ method, which uses region-based and topological characteristics of the image, to guide membership function estimation in FCM.

Let consider $\emptyset(\mathrm{x}, \mathrm{y})$ as the part of interest in $\mathrm{CV}$ results. We define $\mathfrak{C}_{1}=\{\xi \mid \varnothing(\mathrm{x}, \mathrm{y}) \geq 0\}$ and $\mathfrak{C}_{2}=\Omega / \mathbb{C}_{1}$ with cardinality $\tau_{1}$ and $\tau_{2}$ respectively. Thus for every pixel $\xi$, it can be that:

$$
\xi \in \mathbb{C}_{\mathrm{i}} \Leftrightarrow \mathrm{p}\left(\mathfrak{C}_{\mathrm{i}} \mid \xi\right) \geq \mathrm{p}\left(\mathfrak{C}_{\mathrm{j}} \mid \xi\right) \quad \forall \mathrm{i} \neq \mathrm{j}
$$

Now we can compute $\mathrm{p}\left(\mathfrak{C}_{\mathrm{i}} \mid \xi\right)$ using Bayesian rule as follows:

$\mathrm{p}\left(\mathfrak{C}_{\mathrm{i}} \mid \xi\right)=\frac{\mathrm{p}\left(\xi \mid \mathfrak{C}_{\mathrm{i}}\right) \mathrm{P}\left(\mathfrak{C}_{\mathrm{i}}\right)}{\sum_{\mathrm{j}=1}^{2} \mathrm{p}\left(\xi \mid \mathbb{C}_{\mathrm{j}}\right) \mathrm{P}\left(\mathfrak{C}_{\mathrm{j}}\right)} \quad$ for $\mathrm{i}=1,2$

The priors $\mathrm{P}\left(\mathfrak{C}_{\mathrm{i}}\right)$ are estimated based on the proportion of pixels of class $\mathfrak{C}_{i}$. To calculate $p\left(\xi \mid \mathbb{C}_{i}\right)$, we assumed that the distribution generated by the class $\mathfrak{C}_{\mathrm{i}}$ is characterized by a Gaussian kernel as follows:

$\mathrm{p}\left(\xi \mid \mathbb{C}_{\mathrm{i}}\right)=$
$\frac{1}{\left|2 \pi \mathrm{F}_{\mathrm{i}}\right|^{\frac{1}{2}}} \exp \left(-\frac{1}{2}\left(\xi-\mu_{\mathrm{i}}\right)^{\mathrm{T}} \mathrm{F}_{\mathrm{i}}^{-1}\left(\xi-\mu_{\mathrm{i}}\right)\right)$

For $i=1,2$, where $\mu_{i}$ and $F_{i}$ denote the mean value and the covariance matrix of pixels in $\mathbb{C}_{\mathrm{i}}$ respectively.

Therefore, it follows that

$\mathrm{p}\left(\xi \mid \mathfrak{C}_{\mathrm{i}}\right) \mathrm{P}\left(\mathfrak{C}_{\mathrm{i}}\right)=$
$\frac{\tau_{\mathrm{i}}}{\sum_{\mathrm{k}=1}^{2} \tau_{\mathrm{k}}} \frac{1}{\left|2 \pi \mathrm{F}_{\mathrm{i}}\right|^{\frac{1}{2}}} \exp \left(-\frac{1}{2}\left(\xi-\mu_{\mathrm{i}}\right)^{\mathrm{T}} \mathrm{F}_{\mathrm{i}}{ }^{-1}\left(\xi-\mu_{\mathrm{i}}\right)\right)$

The prior membership values of the pixels can be structured in a matrix form as follows:

$$
\aleph=\left\{\left[\aleph_{\mathrm{ij}}\right]_{\mathrm{i}=1,2} \mid \aleph_{\mathrm{ij}}=\mathrm{p}\left(\mathfrak{C}_{\mathrm{i}} \mid \xi_{\mathrm{j}}\right)\right\}
$$

To improve the performance of FCM, the prior memberships degree $N$ which is given by (12) is used in the objective function (6) as follows:

$\mathrm{J}_{\mathrm{fcm}-\mathrm{cv}}(\mathrm{u}, \mathrm{v})=$ 
$\sum_{\mathrm{i}=1}^{2} \sum_{\mathrm{j}=1}^{\mathrm{n}} \mathrm{u}_{\mathrm{ij}}^{\mathrm{m}}\left(\left\|\xi_{\mathrm{j}}-\mathrm{v}_{\mathrm{i}}\right\|^{2}+\gamma \sum_{c \in \in \zeta_{\mathrm{i}}} \boldsymbol{N}_{\mathrm{c} j \mathrm{j}}{ }^{\mathrm{m}}\right)$

in which $C_{i}=\{\{1,2\} \backslash i\}$ and $\gamma$ is a control parameter which keeps a balance between the original FCM and CV method in the optimization procedure.

To find the optimal values of $v_{i}$ and $u_{i j}$, considering the constraint (7), the Lagrange multiplier $\lambda$ is introduced and the following Lagrange function $\mathcal{L}$ is minimized as follows:

$$
\begin{aligned}
\mathcal{L}(U, V, \lambda)=\sum_{i=1}^{2} & \sum_{j=1}^{n} u_{i j}^{m}\left\|\xi_{j}-v_{i}\right\|^{2}+\gamma u_{i j}^{m} \sum_{c \in \zeta_{i}} \aleph_{c j}{ }^{m} \\
& -\lambda\left(\sum_{i=1}^{\eta} u_{i j}-1\right)
\end{aligned}
$$

Assume that $u_{k j}$ is the membership degree of $\xi_{j}$ belonging to the cluster $\eta_{k}$ whose centroid is $v_{k}$. The stationary point of the optimized functional can be obtained as $\left(\mathrm{u}_{\mathrm{kj}}, \mathrm{v}_{\mathrm{k}}, \lambda\right)$ if and only if $\frac{\partial \mathcal{L}}{\partial \lambda}=0, \frac{\partial \mathcal{L}}{\partial \mathrm{v}_{\mathrm{k}}}=0$ and $\frac{\partial \mathcal{L}}{\partial \mathrm{u}_{\mathrm{kj}}}=0$. Taking these derivatives returns the relationships as follows:

$$
\begin{gathered}
\frac{\partial \mathcal{L}}{\partial \lambda}=\sum_{\substack{i=1 \\
n}}^{2} u_{k j}-1=0 \\
\frac{\partial \mathcal{L}}{\partial v_{k}}=2 \sum_{j=1}^{m} u_{k j}^{m}\left(\xi_{j}-v_{k}\right)=0
\end{gathered}
$$

The optimal solution for $\mathrm{v}_{\mathrm{k}}$ is given as follows:

$$
\mathrm{v}_{\mathrm{k}}=\frac{\sum_{\mathrm{j}=1}^{\mathrm{n}} \mathrm{u}_{\mathrm{kj}}^{\mathrm{m}} \xi_{\mathrm{j}}}{\sum_{\mathrm{j}=1}^{\mathrm{n}} \mathrm{u}_{\mathrm{kj}}^{\mathrm{m}}}
$$

Similarly, the derivative of $\mathcal{L}\left(\mathrm{u}_{\mathrm{kj}}\right)$ is taken with respect to $\mathrm{u}_{\mathrm{ij}}$ and fixed it to zero

$$
\begin{aligned}
& \frac{\partial \mathcal{L}}{\partial u_{\mathrm{kj}}}=m u_{\mathrm{kj}}^{\mathrm{m}-1}\left\|\xi_{\mathrm{j}}-\mathrm{v}_{\mathrm{k}}\right\|^{2}+\gamma m u_{\mathrm{kj}}^{\mathrm{m}-1} \sum_{c \epsilon \epsilon_{\mathrm{k}}} \boldsymbol{N}_{\mathrm{c} j}{ }^{\mathrm{m}}- \\
& \lambda=0 \\
& m u_{k j}^{m-1}\left\|\xi_{j}-v_{k}\right\|^{2}+\gamma m u_{k j}^{m-1} \sum_{c \in \zeta_{j}} \kappa_{c j}^{m}=\lambda
\end{aligned}
$$

We set $\mathrm{m}=2$ thus

$\mathrm{u}_{\mathrm{kj}}=\left(\frac{\lambda}{2\left(\left\|\xi_{\mathrm{j}}-\mathrm{v}_{\mathrm{k}}\right\|^{2}+\gamma \sum_{c_{c} \epsilon_{\mathrm{k}}}{N_{c j}}^{2}\right)}\right)$

$\mathrm{u}_{\mathrm{kj}} \in \mathcal{M}$ indicates that

$1=\frac{\lambda}{2} \sum_{\mathrm{i}=1}^{2}\left(\frac{1}{\left(\left\|\xi_{\mathrm{j}}-\mathrm{v}_{\mathrm{i}}\right\|^{2}+\gamma \sum_{c \in \epsilon_{\mathrm{i}}}{\aleph_{c j}}^{2}\right)}\right)$

Hence

$$
\lambda=\frac{2}{\sum_{\mathrm{i}=1}^{2}\left(\frac{1}{\left(\left\|\xi_{\mathrm{j}}-\mathrm{v}_{\mathrm{i}}\right\|^{2}+\gamma \sum_{\varphi \in C_{\mathrm{i}}}{\aleph_{\mathrm{j} j}}^{2}\right)}\right)}
$$

Therefore, the optimal solution of $\mathrm{u}_{\mathrm{kj}}$ is given by:

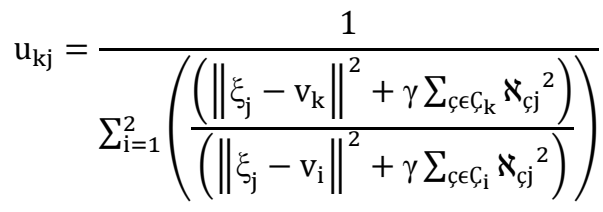

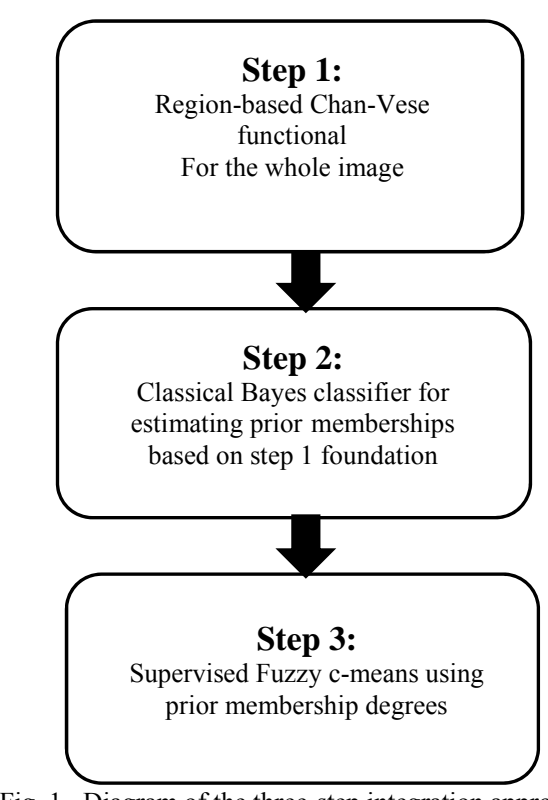

Fig. 1. Diagram of the three-step integration approach.

\section{EXPERIMENTAL RESULTS}

In recent years, there has been significant development in molecular engineering and imaging methodology. The application of high-throughput imaging is of increasing importance in the study of cell systems.

In this section, HT images are used to compare the performance of the proposed algorithm with some wellknown methods, namely conventional FCM [10], CV [4], spatial fuzzy clustering (SFCM) [13], fuzzy level set algorithm (FLS) [14] and region-scalable fitting energy (RSFE) [15]. The data set used is the images of human HT29 colon cancer cells with a size of 512 by 512 pixels from the image set BBBC008v1 [16, 17] which were stained cell nuclei. An example is shown in Fig.1. The ground truth is available for this data set. To evaluate the performance of the algorithm, we used MATLAB to implement all mentioned methods and calculate the misclassification rate (MCR) as follows

$$
\operatorname{MCR}(\%)=\frac{\mathrm{T}_{\text {cell }}+\mathrm{T}_{\text {background }}}{\mathrm{n}} \times 100
$$

where $\mathrm{n}$ indicates the total number of pixels in the given image, $\mathrm{T}_{\text {cell }}$ and $\mathrm{T}_{\text {background }}$ are the number of pixels 
incorrectly classified as the foreground and background respectively.

The misclassification rates for both normal and noisy image are reported in Table I and II. As can be seen the MCR\% values for the integration strategy for all test images were lower than other evaluated methods. The proposed method has consistently superior performance than the other techniques evaluated.

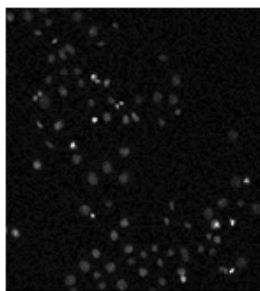

(a)

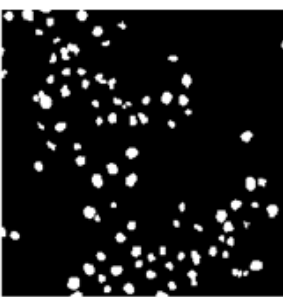

(b)

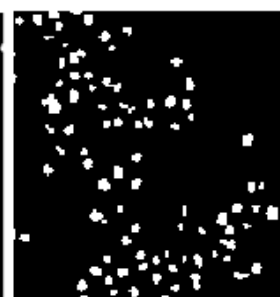

(c)
Fig. 2. Example of (a) Original image, (b) Ground truth and (c) Segmentation result

TABLE I. HUMAN HT29 COLON CANCER CELLS

\begin{tabular}{ccccccc}
\hline $\begin{array}{c}\text { Image } \\
\text { index }\end{array}$ & FCM & CV & SFCM & RSFE & SFLS & $\begin{array}{c}\text { Proposed } \\
\text { Method }\end{array}$ \\
\hline \hline & & & & & & \\
1 & 2.43 & 4.03 & 3.2 & 3 & 2.1 & $\mathbf{0 . 7 5}$ \\
2 & 2.39 & 3.21 & 3.34 & 3.07 & 2.02 & $\mathbf{0 . 6 7}$ \\
3 & 1.64 & 3.12 & 2.17 & 2.05 & 1.41 & $\mathbf{0 . 5 1}$ \\
4 & 0.94 & 1.08 & 1.55 & 1.41 & 1.05 & $\mathbf{0 . 8 1}$ \\
5 & 3.2 & 5.56 & 7.6 & 8.34 & 3.01 & $\mathbf{2 . 8}$ \\
6 & 5.37 & 3.055 & 3.32 & 3.04 & 2.89 & $\mathbf{2 . 3}$ \\
7 & 1.63 & 2.01 & 2.23 & 2.07 & 2.22 & $\mathbf{1 . 4 2}$ \\
8 & 1.16 & 1.45 & 1.7 & 1.71 & 1.57 & $\mathbf{0 . 8 1}$ \\
9 & 1.19 & 1.39 & 1.59 & 1.5 & 1.4 & $\mathbf{0 . 7 8}$ \\
10 & 5.6 & 2.18 & 2.24 & 2.24 & 2.17 & $\mathbf{1 . 6}$ \\
11 & 1.15 & 1.23 & 1.33 & 1.33 & 1.18 & $\mathbf{0 . 8 2}$ \\
12 & 1.47 & 1.5 & 1.77 & 1.79 & 1.45 & $\mathbf{0 . 8 5}$ \\
\hline
\end{tabular}

TABLE II. HUMAN HT29 COLON CANCER CELLS WITH 5\% GAUSSIAN

\begin{tabular}{|c|c|c|c|c|}
\hline \multicolumn{5}{|c|}{ NOISE ADDED } \\
\hline $\begin{array}{l}\text { Image } \mathrm{FCM} \\
\text { index }\end{array}$ & $\mathrm{CV}$ & SFCM RSFE & SFLS & $\begin{array}{l}\text { Proposed } \\
\text { Method }\end{array}$ \\
\hline
\end{tabular}

\begin{tabular}{lllllll}
\hline \hline & & & & & & \\
1 & 4.68 & 5.03 & 4.2 & 3.68 & 4.1 & $\mathbf{1 . 9 9}$ \\
2 & 5.39 & 4.53 & 4.14 & 3.92 & 3.87 & $\mathbf{3 . 0 2}$ \\
3 & 4.64 & 3.52 & 3.07 & 3.21 & 3.15 & $\mathbf{1 . 9 1}$ \\
4 & 1.94 & 2.08 & 1.75 & 1.82 & 1.71 & $\mathbf{1 . 2 5}$ \\
5 & 7.54 & 7.71 & 6.86 & 4.32 & 7.34 & $\mathbf{4 . 3 3}$ \\
6 & 6.46 & 4.53 & 5.17 & 3.94 & 4.12 & $\mathbf{3 . 4 1}$ \\
7 & 2.91 & 3.04 & 2.65 & 2.39 & 2.53 & $\mathbf{2 . 2 1}$ \\
8 & 1.92 & 1.85 & 1.77 & 1.87 & 1.8 & $\mathbf{1 . 3 7}$ \\
9 & 2.38 & 1.99 & 1.83 & 1.8 & 1.81 & $\mathbf{1 . 4 8}$
\end{tabular}

\begin{tabular}{lrrrrrr}
10 & 5.6 & 4.89 & 4.91 & 4.7 & 4.81 & $\mathbf{4 . 0 7}$ \\
11 & 3.13 & 2.8 & 2.54 & 2.42 & 2.51 & $\mathbf{2 . 3 4}$ \\
12 & 2.5 & 2.29 & 2.47 & 2.55 & 2.33 & $\mathbf{1 . 8 8}$ \\
\hline
\end{tabular}

\section{CONCLUSION}

Novel integration strategy which is able to use topological and pixel information of the images to perform image segmentation was introduced. A three-step approach is presented. First, the CV level set method, which is able to handle topological features of the images, is used. Then, Bayes classifier is utilized to estimate prior membership values based on the first step segmentation. Finally a novel FCM based objective function is implemented, which uses pixel and topological information given by the prior at the same time, to perform pixel foreground-background classification. Experiments on a benchmark set of colon cancer cell images show that the proposed integration strategy can attain more accurate segmentations.

A disadvantage of the proposed method is its computational cost, which is mainly dependent on the individual methods in the integration framework. In addition, how to determine the optimal control parameter $\gamma$ adaptively according to each image to guarantee an accurate segmentation will be our future work.

\section{ACKNOWLEDGMENT}

This work was supported by the Australian Research Council (ARC) Discovery Grant DP1097059 and the Griffith International Postgraduate Research Scholarship (GUIPRS).

\section{REFERENCES}

[1] R. Malladi, J. A. Sethian, and B. C. Vemuri, "Shape modeling with front propagation: A level set approach,"IEEE Transaction on Pattern Analysis and Machine Intelligence, vol. 17, no. 2, 1995, pp. 158-175.

[2] V. Caselles, F. Catte, T. Coll, and F. Dibos, "A geometric model for active contours in image processing," Numerische Mathematik, vol. 66, no. 1,1993, pp.1-31.

[3] S. Osher and J. A. Sethian, "Fronts propagating with curvature-dependent speed: Algorithms based on HamiltonJacobi Formulation," Journal of Computational Physics, vol. 79, 1988, pp. 12-49.

[4] T.F. Chan and L.A. Vese, "Active Contours without edges," IEEE transactions on Image Processing, vol. 10, no.2, 2001, pp.266-277.

[5] A.W.C. Liew, S.H. Leung and W.H. Lau, "Fuzzy Image Clustering Incorporating Spatial Continuity", IEEE ProceedingsVision, Image and Signal Processing, vol. 147, no.2 , 2000, pp. 185-192.

[6] A.W.C. Liew and H. Yan, "An Adaptive Spatial Fuzzy Clustering Algorithm for MR Image Segmentation", IEEE Transactions on Medical Imaging, vol. 22, no. 9, 2003, pp. 10631075.

[7] D. Mumford and J. Shah, "Optimal approximation by piecewise smooth functions and associated variational 
problems," Communications on Pure and Applied Mathematics, vol. 42, 1989, pp. 577-685.

[8] J. M. Morel and S. Solimini, "Segmentation of Images by Variational Methods: A Constructive Approach", Madrid, Spain: Revista Matematica Universidad Complutense de Madrid, vol. 1, 1988, pp. 169-182.

[9] J. Dunn, "A fuzzy relative of the ISODATA process and its use in detecting compact, well-separated clusters," Journal of Cybernetics, vol. 3, no. 3, 1973, pp. 32-57.

[10] J. Bezdek, Pattern recognition with fuzzy objective function algorithms, Plenum Press, New York, 1981.

[11] N.R. Pal, K. Pal, J. M. Keller, J. Bezdek, “A possibilistic fuzzy c-means clustering algorithm," IEEE Transactions on Fuzzy Systems, vol. 13, no. 4, 2005, pp. 517-530.

[12] F. Höppner and F. Klawonn, "A contribution to convergence theory of fuzzy c-means and derivatives," IEEE Transactions on Fuzzy Systems, vol. 11, 2003, no. 5, pp. 682-694.

[13] K.S. Chuang, H.L. Hzeng, S. Chen, J. Wu, T.J. Chen, 'Fuzzy c-means clustering with spatial information for image segmentation," Computerized Medical Imaging and Graphics, vol.30, 2000, pp. 9-156.

[14] Li, B. N., Chui, C. K., Chang, S., \& Ong, S. H. , "Integrating spatial fuzzy clustering and level set methods for automated medical image segmentation," Computers in Biology and Medicine, vol. 41, 2011, pp.1-10.

[15] Li, C., Kao, C., Gore, J., Ding, Z., "Minimization of region-scalable fitting energy for image segmentation," IEEE Transactions on Image Processing, vol.17, 2008, pp.1940-1949.

[16] www.broadinstitute.org/bbbc

[17] A.E. Carpenter, T.R. Jones, M.R. Lamprecht, C. Clarke, IH. Kang, O. Friman, DA. Guertin, JH. Chang, RA. Lindquist, J. Moffat, P. Golland, DM. Sabatini, "CellProfiler: image analysis software for identifying and quantifying cell phenotypes," Genome Biology, 7:R100, 2006. 